\title{
ImageJ macros for the user-friendly analysis of soft-agar and wound-healing assays
}

\author{
João Paulo Silva Nunes and Adriana Abalen Martins Dias \\ Laboratory of Experimental Genetics, General Biology Department - ICB, Universidade Federal de Minas \\ Gerais, Belo Horizonte, Brazil
}

BioTechniques 62:175-179 (April 2017) doi 10.2144/000114535

Keywords: macro; ImageJ; wound-healing; soft-agar; cancer

Supplementary material for this article is available at www.BioTechniques.com/article/114535.

Recent advances in biological imaging techniques and the enormous amount of data they generate call for the development of computational tools for efficient and reliable high-throughput analysis. Several software applications with this functionality are available, and one of the most commonly used is ImageJ. Here, we present two independent macros (WH_NJ and SA_NJ) for automating and facilitating the analysis of images acquired from two in vitro assays frequently used in cancer studies and drug screening: the wound-healing and soft-agar assays. These two algorithms combine, in a single command, the steps required for the individual analysis of each image using ImageJ. WH_NJ and SA_NJ allow fast, reproducible data analysis without the experimental bias inherent in manual analyses, thus guaranteeing the robustness and reliability of the results.

The development of computerized methods for large-scale analyses of imaging data are currently needed to fill the gap between the acquisition of hundreds of images by increasingly fast and efficient automated techniques and the subjective evaluation of those images by researchers (1). For instance, microscopy is an important tool for analyzing changes in cell morphology in response to specific agents, but the amount of data generated by automated techniques greatly exceeds manual processing capacity (2).

Several experimental in vitro methods are currently available to evaluate the effects of drugs on the morphological and physiological characteristics of cancer cells (3). The wound-healing assay and the evaluation of anchorageindependent growth in soft-agar are two classic techniques used for screening antitumor drugs $(4,5)$. The first assay evaluates the effects of selected drugs on cell migration, which is a central feature of tumor metastasis $(6,7)$ that allows neoplastic cells to invade the surrounding blood and lymphatic vessels and spread to other organs $(8,9)$. The second assay, on the other hand, is one of the tests that most clearly show the malignant transformation of cells, and it is based in the concept that transformed non-neoplastic cells and tumor cells are able to survive, grow, and form colonies in the absence of anchorage (such as to the extracellular matrix in tissues) or contact with neighboring cells (10). These assays have the potential to provide insights on the effects that drugs may have on the morphological characteristics of tumor cells. Both techniques generate results using microscopy and image processing, in which the morphology of migrating or colony-forming cells is examined in response to specific agents.

In recent years, biological imaging techniques have become increasingly sophisticated and accessible, creating a demand for computational tools capable of processing and analyzing the enormous amount of data generated (11). Indeed, several computational tools have been developed to process biological images, such as Matlab (MathWorks, Inc.) (12),
Image Pro Plus (Media Cybernetics) (13), TScratch (CSElab) (14), ColonyArea (15), and other custom-made algorithms for specific tasks. However, the everincreasing amount of biological images obtained from different experiments has required the further development of tools for processing images able to meet the needs of different tests. Moreover, currently available software applications are not always user-friendly or may be too expensive for many researchers. Thus, there is a need for new automated computational algorithms that perform unbiased, user-friendly analyses of high-throughput data and simplify data processing while guaranteeing robustness and reproducibility of the results at a reasonable cost.

Here, we present two algorithms built using the ImageJ programming language (National Institutes of Health) to facilitate automated analyses of images from cell migration ( $\mathrm{WH} \_\mathrm{NJ}$ algorithm) and anchorage-independent growth in soft-agar (SA_NJ algorithm) assays. We used Image $\mathrm{J}$ since it is a free and opensource application for the processing of

\section{METHOD SUMMARY}

We present two independent algorithms, coded using the ImageJ macro language (IJM), that speed up and eliminate bias in the analysis of phase contrast optic microscopy images obtained during wound-healing and soft-agar assays. 
multidimensional images (16). Moreover, the flexibility of the ImageJ language allows for multiple plugins and macros to be created for a variety of tasks, facilitating statistical calculations (16).

Currently, analyses of cell migration are most frequently performed by manual tracing of two parallel lines bordering the edges of the regions without cells, by outlining the borders of the wound (17), or by using the sizes of straight lines connecting the borders of the wounds (18-20). For the soft-agar assay, the formula commonly employed is $A=\pi R r$, where $\pi=3.14$, and $R$ and $r$ are the longest and the shortest radii, respectively, of the colonies; thus, the researcher must measure the diameter of each colony (21). These techniques are necessarily subjective since the researcher has to manually trace the regions of interest in each image (i.e., areas lacking cells or the areas of the colonies). This is particularly problematic for large-scale drug screening experiments with hundreds of images, when the task becomes laborious and time-consuming (2). The algorithms presented here stand out because they overcome these two problems, allowing precise, rapid, and unbiased analyses of both assays.

\section{Materials and methods}

Cell culture and image acquisition Human melanoma SK-MEL-37 cells were cultured as described in Carey et al. (22). For the wound-healing assay, cells (1.5 $\left.10^{5}\right)$ were plated in technical triplicates in 24-well plates $\left(1.9 \mathrm{~cm}^{2}\right.$ surface area/ well). After reaching 100\% confluence, 2 wounds $\sim 2-\mathrm{mm}$ wide were made in the monolayer with a 200- $\mu$ l sterile pipette tip. Detached cells were removed by washing with sterile 1× PBS (phosphate buffered saline; USB Affymetrix, Santa Clara, CA) pre-warmed to $37^{\circ} \mathrm{C}$. Two independent areas of each lesion were imaged at 0 , 12, 24, 48 and $72 \mathrm{~h}$ with a Moticam 2300 camera attached to a Motic AE31 inverted phase contrast microscope (Motic Asia, Kowloon Bay, Kowloon, Hong Kong) at 40x magnification.

For the soft-agar assay, SK-MEL-37 cells $\left(1.0 \times 10^{3}\right)$ were suspended in $300 \mu$ I DMEM (Dulbecco's Modified Eagle Medium; ThermoFisher Scientific, Waltham, MA) supplemented with 10\% FBS (ThermoFisher Scientific) and 0.3\% ultra-pure agarose (Sigma-Aldrich, St.

A
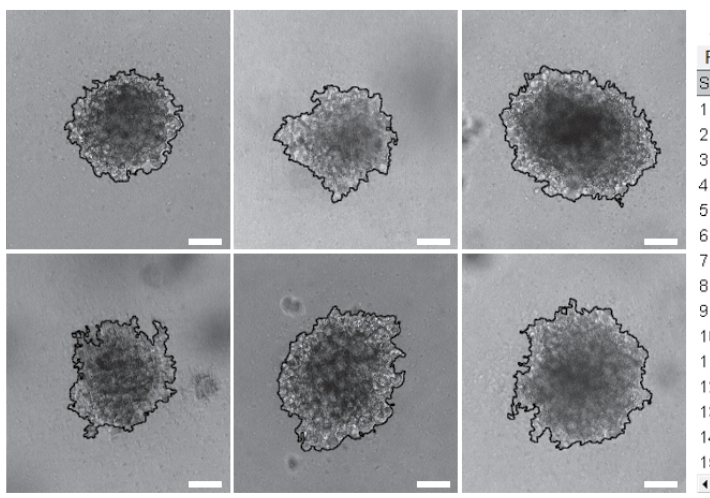

B
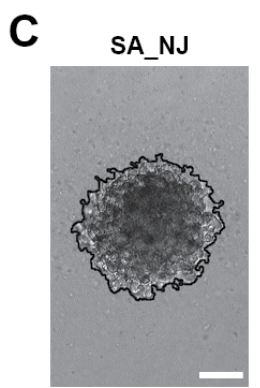

Manual analysis

D
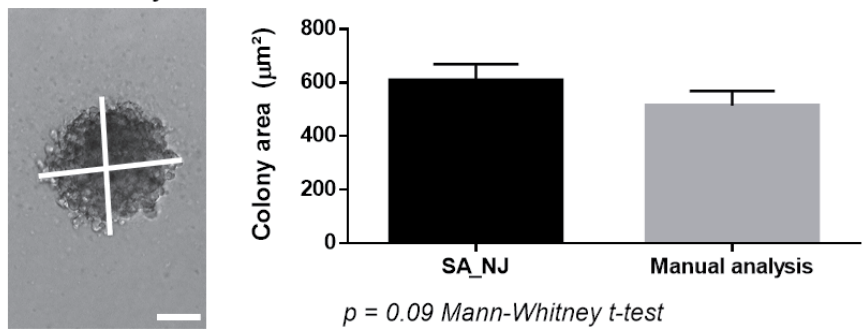

Figure 1. Serial demarcation and calculation of colony area using the SA_NJ macro. The SA_NJ macro developed in this study automatically defines the areas of cell colonies grown in suspension, which are imaged using a phase contrast microscope; the macro generates numerical data corresponding to the area of the colony in relation to the whole image. (A) Delimitation of six colonies with different sizes and shapes (black borders), indicating the regions used by the macro for calculation of area. (B) Window showing a summary of the calculation of the areas of 45 colonies, listing the raw data referring to the number of colonies per image, the total area, the mean size (if there is more than one colony per image), and the percentage of the area of the colony in relation to the total area of the image. (C) Comparison of the calculation of the area of one colony using the SA_NJ macro and the manual method of measuring two diameters ( $A=\pi R r$; $R$ and $r$ are the longest and the shortest radii, respectively). (D) Statistical analysis demonstrating that there was no statistically significant difference in the area measurements using the SA_NJ macro versus the manual method ( $n=45$ colonies). Scale bar: $200 \mu \mathrm{m}$.

Louis, MO). The suspension was poured over $300 \mu \mathrm{l}$ pre-solidified $0.5 \%$ agar base (Sigma-Aldrich) in 24-well plates (1.9 $\mathrm{cm}^{2}$ surface area/well), and the plates were incubated at $37^{\circ} \mathrm{C}$ with $5 \%$ $\mathrm{CO}_{2}$ atmosphere saturation. Cells were cultivated for 3 weeks, and the growth medium on top of the agarose layer was replaced twice a week. Colonies $(n=45)$ were photographed using a Moticam 2300 camera attached to a Motic AE31 inverted phase contrast microscope at 100x magnification.

In both experiments, images of the cells were captured in JPEG format using the Motic Image Plus 2.0 software for Microsoft Windows XP (Redmond, WA). This software captures images of cells visualized on the Motic AE31 inverted phase contrast optic microscope attached to a Moticam 2300 camera. Photograph parameters were optimized to obtain high resolution (i.e., $2048 \times 1536$ pixels) and sharpness in the image, and for the best balance of brightness and contrast that allows for clear distinction of regions with and without cells (Supplementary Figure S1).

\section{Image processing}

We used two online open-access software applications to calculate the areas of wounds in the cell migration assay and the sizes of colonies grown in the soft-agar assay: (i) the FastStone Image Viewer 5.7 (www.faststone.org/FSViewerDownload. htm), which clusters images of replicates and/or treatment groups, generating a single file for analysis; and (ii) ImageJ version 1.49, which can be obtained from the RSB home page (https://imagej.nih. gov/ij/). The subsequent steps involving the ImageJ macros were executed and tested in the following operating systems: Microsoft Windows versions 7, 8, 8.1, and 10, and Ubuntu 16.04.1 LTS, instructions set $\times 64$ and $\times 86$.

For the installation of both the $\mathrm{WH}_{-}$ NJ and SA_NJ algorithms in Windows, ImageJ needs to be executed and 
calibrated (Supplementary Figure S2). The next steps are as follows: click on the menu item "Plugins," sub-menu "Macros," "Install," and in the directory of macros available, select the one of interest and click "Open." After restarting the software, a tab associated with each macro should be available in the ImageJ window under "Plugins."

Optionally, a file with multiple images can be created for an automated analysis of several images. To this end, the following instructions must be executed: open the FastStone Image Viewer, go to the directory where the images are stored, select the photographs of interest (e.g., a control group), go to the menu "Create" and click on "Multi-Page File Builder"(or just press Alt $+M)$. In the new window, choose the TIFF format, select "UNCOMPRESSED" in the option "Compression," and click on "Create." Name the file, choose a destination folder, and click on "Save." Additionally, the user can opt to open all images using the native option available in ImageJ instead of creating a stacked image (Supplementary Figure S3).

After creation of the file and installation of the macros, open the images in Image J and execute the algorithm corresponding to the experiment that will be analyzed. The time spent calculating the areas varies according to the number of images. After the calculation, three new windows will open in ImageJ: (i) ROI Manager, which stores the information about the overlay; (ii) the results window, which shows the areas calculated for each region identified in the image; and (iii) the summary of the results.

\section{Results and discussion}

The SA_NJ macro allowed the calculation of the areas of 45 independent colonies of tumor cells grown in suspension in soft agar, with just a single click. This algorithm distinguishes the pixels in the regions with and without cells in the images, precisely identifying the entire extent of the colony. The SA_NJ macro then automatically creates a line surrounding the colonies, clearly defining the region to be used by the macro for calculation of the areas (Figure 1A). Next, ImageJ generates values for each colony, which are summarized in a new window (Figure 1B) showing the area of each colony and the proportion it represents of the whole image.

The mean area calculated by SA_NJ $\left(607 \pm 59.9 \mu \mathrm{m}^{2}\right)$ showed no statistically significant difference when compared with the mean area calculated using the manual method of measuring the longest and shortest radii of the colonies and

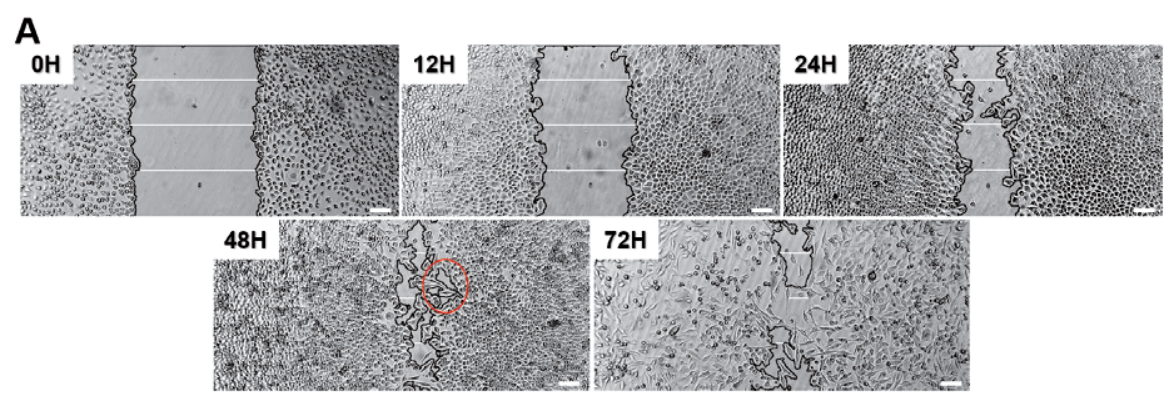

B

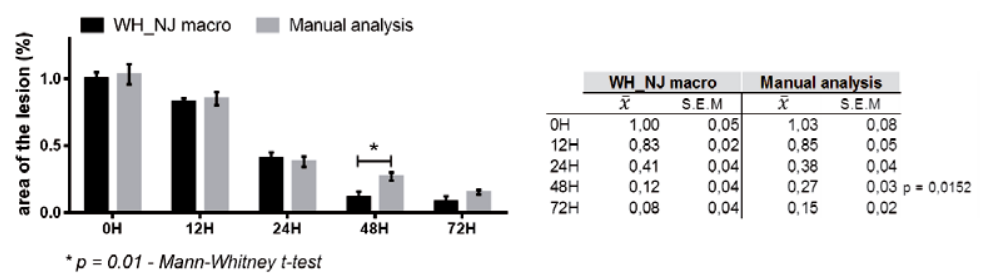

Figure 2. Use of the ImageJ WH_NJ macro for analysis of the wound-healing cell migration assay. Regeneration of a lesion made in the cellular monolayer at time $0 \mathrm{~h}(\mathrm{H})$ was evaluated $12,24,48$, and $72 \mathrm{~h}(\mathrm{H})$ after the wound was made. (A) The WH_NJ macro was performed for each time point, and the regions with and without cells were identified (demarcated by the black line) and used for the calculation of the area of the wound. Manual analysis was performed by drawing three parallel lines connecting the edges of the wound for each image (white lines), and the mean length of the lines was used to determine the size of each wound. (B) Graph derived from data from six technical replicates (WH_NJ) and three parallel lines (manual analysis) for each sample showing the percentage of the area of the lesion in relation to the initial $(\mathrm{OH})$ total area over time. The table shows individual mean $\left(x^{-}\right)$and standard error of the mean (SEM) values obtained by the two measurement methods. Red circle shows the region omitted from manual measurement. Scale bar: $500 \mu \mathrm{m}$.

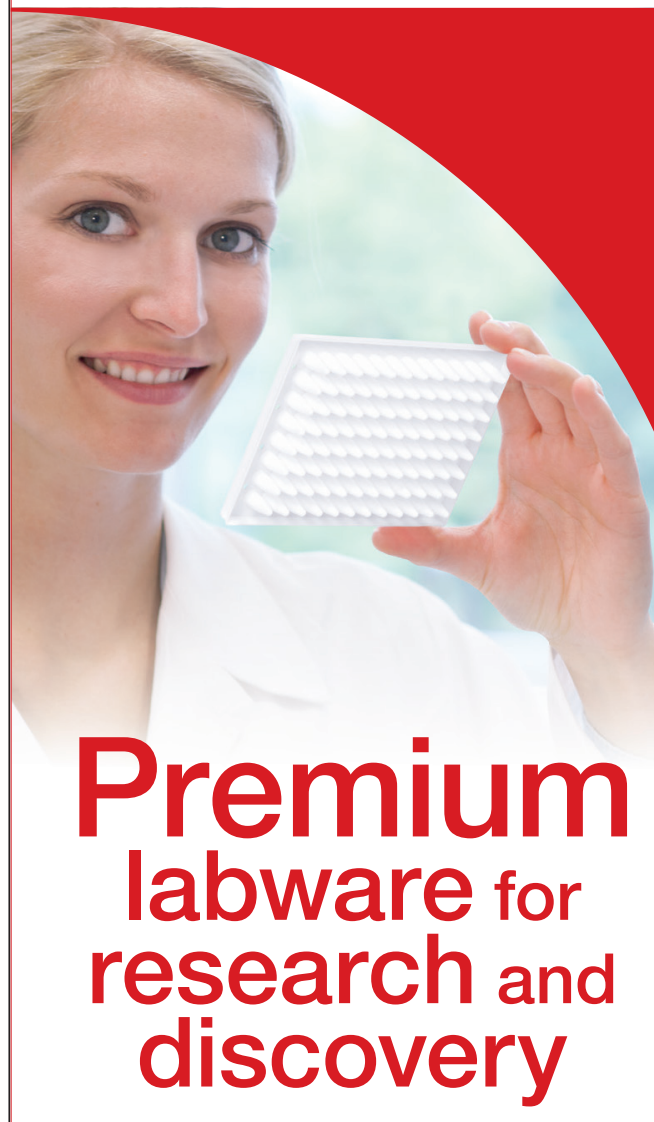

\section{Certified qPCR Consumables}

- Full range of plate styles and 8-strip tubes

-White wells maximize signal reflection

- Optically clear lid strips and sealing tapes
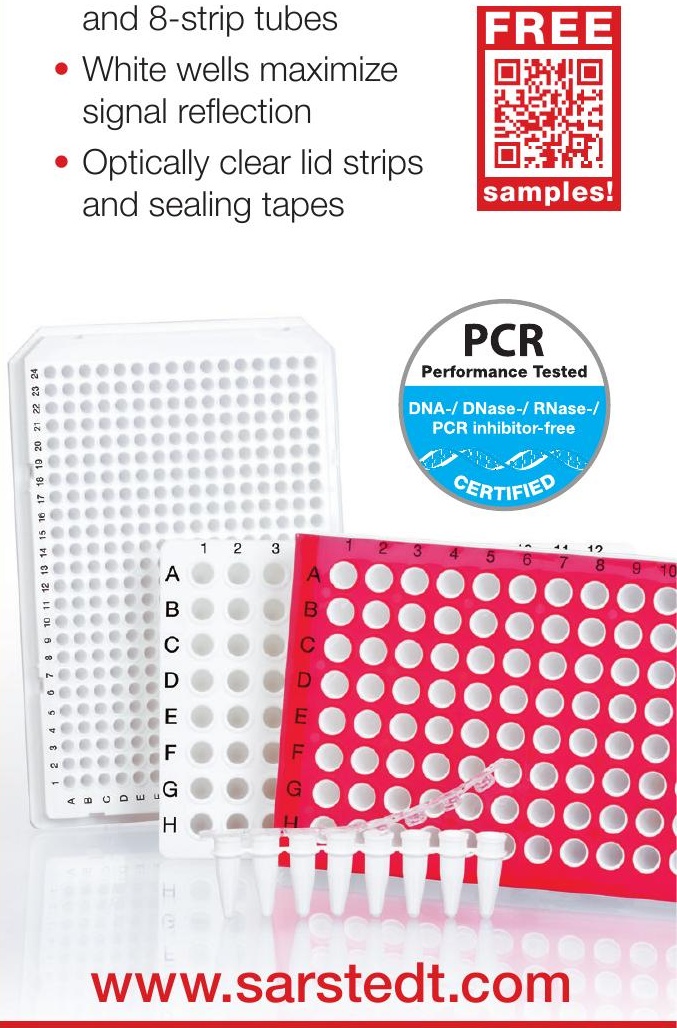


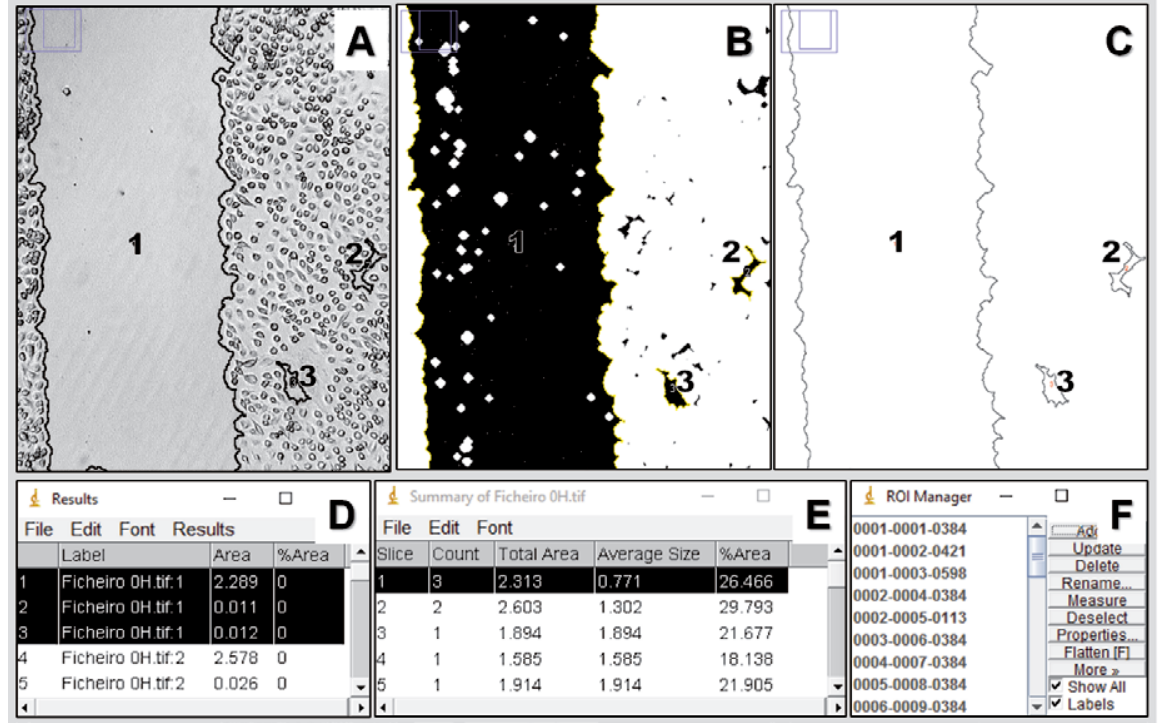

Figure 3. Wound-healing assay data obtained after calculation in Image J. The WH_NJ macro was validated by evaluating its precision in the detection and calculation of areas related to wounds made in the cell monolayer, using >300 images. (A) The WH_NJ macro for cell migration assays calculates the area of the wound (A1), but can also detect regions without cells and calculate their areas (A2 and A3). In these cases, the researcher will need to collect data only from the area of the lesion (A1). (B) Automatic conversion of the image in Panel A for calculation of the area. (C) Automatically generated lines that are used to overlay the original image. In these cases, the researcher will need to collect data from the area of the wound (D) only in the window showing the areas of each identified region (including the wound). (E) Summary window in which the information from all of the areas detected in the image are gathered. The percentages of the areas of all of these regions in relation to the total area of the image is shown. (F) ROI Manager, where information regarding the demarcation lines is stored.

applying the formula $A=\pi \operatorname{Rr}(21)(513$ $\pm 53.85 \mu \mathrm{m}^{2}$ ) (Figures 1, C and D). This demonstrates that SA_NJ is accurate in measuring the areas of the colonies, with the advantage of being a one-click method. Moreover, the manual method presents additional drawbacks. In some cases, the identification of the longest and shortest diameters is compromised by the morphology of the colony (which is frequently not oval) and requires two or more researchers to perform independent measurements of each colony, making the analysis laborious and time-consuming.

In the wound-healing assay, we employed two methods. The first involved measuring the lengths of three parallel lines connecting the borders of each scratch and using their mean to estimate the size of the lesion (white lines of Figure $2 \mathrm{~A}$ ), whereas the second method used the WH_NJ macro (black lines of Figure 2A). $\mathrm{WH} \_\mathrm{NJ}$ automatically identified the regions without cells (i.e., the wound) and calculated the ratio of this area to the area of the whole image over time (Figure 2A). Our macro showed statistical consistency, as demonstrated by the small standard errors of the mean obtained from the technical triplicates as well as the similarity of the histogram showing closure of the lesion over time to that obtained using the manual method (Figure 2B). The WH_NJ macro also precisely calculated areas uncovered by the cells that were skipped when using the manual method (red circle in Figure 2A). The high accuracy and precision of the WH_NJ macro affected the results, as demonstrated when wound regeneration at $48 \mathrm{~h}$ was compared between the 2 methods: $12 \pm 4 \%$ for $\mathrm{WH}$ _NJ and 27 $\pm 3 \%$ for the manual method; $P=0.0152$ (Figure 2B, 48H). The other advantage of this macro in comparison to other methods (23) is that it specifically detects the regions of the wound in an automated and unbiased way.

In some analyses, however, the sensitivity of the WH_NJ macro can detect holes in areas that are not of interest (Figure 3). In these cases, it is important that the users make an overlay of the line generated by Image J (Figure 3, A and B) and the original image. For that, the following steps have to be executed: without closing any window, re-open the original figure (used for the calculation) using ImageJ and go to the menu "Image," option "Overlay," and click on "From ROI Manager" (Figure 3F). The line generated in Figure 3B will be incorporated into the original image (Figure $3 C)$, creating an overlay. In this example,
3 regions were identified, and their respective areas were calculated (Area 1: 2.289 $\mu \mathrm{m}^{2}$, Area 2: $0.011 \mu \mathrm{m}^{2}$, and Area 3: $0.012 \mu \mathrm{m}^{2}$ ) (Figure 3D). In this case, the researcher will have to disregard the Area 2 and Area 3 values when copying the data to a spreadsheet for statistical analysis. In Figure 3E, the sum of the areas calculated for the three regions identified (Area 1, Area 2 , and Area 3 ) is indicated, as well as the mean of these areas and the percentage that these areas represent in relation to the whole image.

Alternatively, it is possible to configure the software to automatically disregard smaller areas using the following steps: close windows $B, C, D$, and $F$ shown in Figure 3, and go to the menu "Analyze," and "Analyze particles." Keep the options "Display results," "Summarize," and "Add to manager" selected, and in "Size," gradually increase the value by 0.05 points to discard smaller areas. Click OK, and repeat the process of composition to check if the new settings applied are satisfactory. It is worth noting that the established value should be the same for all of the groups analyzed in a given experiment.

ImageJ has the advantage of being a freely distributed software; however, many of its functionalities are not easily appreciated by users, and require basic understanding of computer programming. As a consequence, ImageJ is not always the researchers' first choice for image analysis, and many laboratories opt for commercial tools or outsourced services. Both the WH_NJ and the SA_NJ macros were designed with the aim of circumventing this problem and facilitating analyses of wound-healing and soft-agar assays. They allow for the systematic measurement and analysis of photographs of cell cultures in an automated and reproducible way. Moreover, they are easy to use, require minimal user input, and present numerous advantages relative to other methods currently in use. Additionally, these macros are able to perform large-scale automated analyses of an infinite amount of images, which is not possible using some methods published to date $(17,19,24,25)$, These macros also minimize manual intervention and eliminate the need for expensive and robust fluorescence microscopy tools, or commercial software for image processing, such as Image-Pro Plus (13). A previous study compared the use of Matlab and ImageJ 
for assessing the migration rate from an in vitro circular model. The authors wanted highly precise measurements of singlecell migration events from one image at a time and showed that ImageJ generated data with similar robustness to Matlab (12). However, despite being a complete software application that performs many types of biological and statistical analyses, Matlab is a costly and highly complex program that needs to be mastered by the user and requires constant Internet connection. Other free applications used to evaluate the size of scratches (e.g., TScratch, a Matlab derivative) (14) and the area of colonies (e.g., ColonyArea) (15) also require that the user input many parameters, instead of being single-click. Although these applications are powerful and efficient tools, they are not as easy to use as the macros presented here.

The current work presents two computational macros that provided similar results with simpler usage than other comparable software tools. The macros presented here were validated by a comparison with corresponding manual methods and have proven to be ideal for users seeking a simple, efficient, and reproducible way to calculate the pattern of migration and colony formation for their assays.

Both the WH_NJ and SA_NJ macros are available for download from the database of macros at the ImageJ web site, or they can be requested by e-mailing the authors. Both files are available in the formats ".ijm" and ".txt." While the original raw code of the macros is presented in Supplementary Figure S4, they will be updated as requested by users in order to address specific demands.

\section{Author contributions}

A.A.M.D. contributed to the conception and design of the work, performed data analysis interpreted results, performed critical revision of the manuscript, and approved the final version of the manuscript. J.P.S.N. contributed to the conception and design of the work, development of algorithms, data collection, analysis, and interpretation, drafting of the article, and approval of the final version of the manuscript.

\section{Competing interests}

The authors declare no competing interests.

\section{Acknowledgments}

We thankErna Geessien Kroon and Jônatas Abrahão from the Virology Laboratory (Instituto de Ciências Biológicas/UFMG) for the use of the cell culture and imaging facilities. We are grateful to Priscila Fabiana Rodrigues for testing the macro in several experiments, to Joyce Esposito de Souza for revision of the manuscript, and to CNPq, CAPES, FAPEMIG, and Pro-reitoria de Pesquisa/UFMG for the financial support given to this research.

\section{References}

1. Wollman, R. and N. Stuurman. 2007. High throughput microscopy: from raw images to discoveries. J. Cell Sci. 120:3715-3722.

2. Chen, W., C. Wong, E. Vosburgh, A.J. Levine, D.J. Foran, and E.Y. Xu. 2014. High-throughput Image Analysis of Tumor Spheroids: A Userfriendly Software Application to Measure the Size of Spheroids Automatically and Accurately. J. Vis. Exp. (89):51639.

3. Lovitt, C.J., T.B. Shelper, and V.M. Avery. 2014. Advanced Cell Culture Techniques for Cancer Drug Discovery. Biology (Basel) 3:345367.

4. Kam, Y., C. Guess, L. Estrada, B. Weidow, and V. Quaranta. 2008. A novel circular invasion assay mimics in vivo invasive behavior of cancer cell lines and distinguishes single-cell motility in vitro. BMC Cancer 8:198.

5. Rotem, A., A. Janzer, B. Izar, Z. Ji, J.G. Doench, L.A. Garraway, and K. Struhl. 2015. Alternative to the soft-agar assay that permits high-throughput drug and genetic screens for cellular transformation. Proc. Natl. Acad. Sci. USA 112:5708-5713.

6. Friedl, P. and K. Wolf. 2003. Tumour-cell invasion and migration: diversity and escape mechanisms. Nat. Rev. Cancer 3:362-374.

7. Sahai, E. and C.J. Marshall. 2003. Differing modes of tumour cell invasion have distinct requirements for $\mathrm{Rho/ROCK}$ signalling and extracellular proteolysis. Nat. Cell Biol. 5:711-719.

8. Mattila, P.K. and P. Lappalainen. 2008. Filopodia: molecular architecture and cellular functions. Nat. Rev. Mol. Cell Biol. 9:446-454.

9. Nguyen, T. and R.M. Mège. 2016. N-cadherin and fibroblast growth factor receptors crosstalk in the control of developmental and cancer cell migrations. Eur J Cell Biol. 95:415-426.

10. Borowicz, S., M. Van Scoyk, S. Avasarala, M.K. Karuppusamy Rathinam, J. Tauler, R.K. Bikkavilli, and R.A. Winn. 2014. The soft agar colony formation assay. J. Vis. Exp. (92):e51998.

11. Barry, D.J., C.H. Durkin, J.V. Abella, and M. Way. 2015. Open source software for quantification of cell migration, protrusions, and fluorescence intensities. J. Cell Biol. 209:163-180.

12. Treloar, K.K. and M.J. Simpson. 2013. Sensitivity of edge detection methods for quantifying cell migration assays. PLoS One 8:e67389.

13. Yarrow, J.C., Z.E. Perlman, N.J. Westwood, and T.J. Mitchison. 2004. A high-throughput cell migration assay using scratch wound healing, a comparison of image-based readout methods. BMC Biotechnol. 4:21.
14. Gebäck, T., M.M. Schulz, P. Koumoutsakos, and M. Detmar. 2009. TScratch: a novel and simple software tool for automated analysis of monolayer wound healing assays. Biotechniques 46:265-274.

15. Guzmán, C., M. Bagga, A. Kaur, J. Westermarck, and D. Abankwa. 2014. ColonyArea: An ImageJ plugin to automatically quantify colony formation in clonogenic assays. PLoS One. 9:e92444.

16. Schneider, C.A., W.S. Rasband, and K.W. Eliceiri. 2012. NIH Image to ImageJ: 25 years of image analysis. Nat. Methods 9:671-675.

17. Ferreira, A.K., V.M. Freitas, D. Levy, J.L. Ruiz, S.P. Bydlowski, R.E. Rici, O.M. Filho, G.O. Chierice, and D.A. Maria. 2013. Anti-angiogenic and anti-metastatic activity of synthetic phosphoethanolamine. PLoS One. 8:e57937.

18. Li, Y., C. Zeng, M. Tu, W. Jiang, Z. Dai, Y. Hu, Z. Deng, and W. Xiao. 2016. MicroRNA-200b acts as a tumor suppressor in osteosarcoma via targeting ZEB1. Onco Targets Ther. 9:3101-3111.

19. David, D., S. Jagadeeshan, R. Hariharan, A.S. Nair, and R.M. Pillai. 2014. Smurf2 E3 ubiquitin ligase modulates proliferation and invasiveness of breast cancer cells in a CNKSR2 dependent manner. Cell Div. 9:2.

20. Jiang, F., T. Liu, Y. He, Q. Yan, X. Chen, H. Wang, and X. Wan. 2011. MiR-125b promotes proliferation and migration of type II endometrial carcinoma cells through targeting TP53INP1 tumor suppressor in vitro and in vivo. BMC Cancer 11:425.

21. Rodrigues, D.F., F.F. Mendes, T.A. Dias, A.R.d. Lima, and L.A.F.d. Silva. 2013. O programa ImageJ como ferramenta de análise morfométrica de feridas cutâneas. Enciclopédia Biosfera. 9:1955-1963.

22. Carey, T.E., T. Takahashi, L.A. Resnick, H.F. Oettgen, and L.J. Old. 1976. Cell surface antigens of human malignant melanoma: mixed hemadsorption assays for humoral immunity to cultured autologous melanoma cells. Proc. Natl. Acad. Sci. USA 73:3278-3282.

23. Ho, B.Y., C.H. Lin, M.K. Apaya, W.W. Chao, and L.F. Shyur. 2012. Silibinin and Paclitaxel Cotreatment Significantly Suppress the Activity and Lung Metastasis of Triple Negative 4T1 Mammary Tumor Cell in Mice. J. Tradit. Complement. Med. 2:301-311.

24. Wu, W., J. Yang, X. Feng, H. Wang, S. Ye, P. Yang, W. Tan, G. Wei, and Y. Zhou. 2013. MicroRNA-32 (miR-32) regulates phosphatase and tensin homologue (PTEN) expression and promotes growth, migration, and invasion in colorectal carcinoma cells. Mol. Cancer 12:30.

25. Aguiar, C., J. Therrien, P. Lemire, M. Segura, L.C. Smith, and C.L. Theoret. 2016. Differentiation of equine induced pluripotent stem cells into a keratinocyte lineage. Equine Vet. J. 48:338345.

Received 16 November 2016; accepted 30 January 2017.

Address correspondence to: Adriana Abalen Martins Dias, Instituto de Ciências Biológicas, Departamento de Biologia Geral, Universidade Federal de Minas Gerais, Av. Antônio Carlos, 6627, Pampulha. Belo Horizonte, MG, Brasil - 31270-901. E-mail: abalen@ufmg.br

To purchase reprints of this article, contact: biotechniques@fosterprinting.com 